\title{
THERMAL EXPANSION ANOMALIES AT SPIN-REORIENTATION IN SmFe THE ROLE OF THE MAGNETIC TEXTURE
}

\author{
S.M. PODGORNYKH*, A.V. KOROLYOV, V.S. GAVIKO \\ and N.V. MUSHNIKOV \\ Institute of Metal Physics, Ural Branch of RAS, Kovalevskaya 18, \\ 620219 Ekaterinburg, Russia
}

(Received in final form 28 September 1997)

Temperature dependency of the thermal expansion coefficient (TEC) was studied at 4.2$300 \mathrm{~K}$ in $\mathrm{SmFe}_{2}$ ferromagnetic compound having spin-reorientation temperature near $190 \mathrm{~K}$. We have found a strong effect of the magnetic texture on TEC of this high magnetostrictive compound at the spin-reorientation phase transition from [ $\left.\begin{array}{lll}1 & 1 & 1\end{array}\right]$ to [ $\left[\begin{array}{lll}1 & 1 & 0\end{array}\right]$ easy direction. The substantial role in the texture formation is expected to belong to atoms of hydrogen that are always present in a small amount in the compounds of such type.

Keywords: Thermal expansion; Spin-reorientation phase transition; Magnetic texture; $\mathrm{SmFe}_{2}$

\section{INTRODUCTION}

According to the Akulov's rule of even effects, the anisotropic linear magnetostriction $(\lambda)$ does not contribute to the thermal expansion of a polycrystalline polydomain specimen. That is, in the first approximation, a rotation of the magnetization, for example at spin-reorientation transition, does not give rise to any volume magnetostriction. However, when a magnetic texture is created in a specimen with high $\lambda$ value, a deformation due to magnetostriction may be observed by thermal expansion of the specimen with pseudocubic structure. We have found a strong effect of the magnetic texture on the thermal expansion of high

* Corresponding author. 
TABLE I Magnetostriction constants of $\mathrm{SmFe}_{2}$

\begin{tabular}{lcccccc}
\hline Parameter & \multicolumn{6}{c}{ Temperature (K) } \\
\cline { 2 - 7 } & 4.2 & 100 & 150 & 200 & 250 & 300 \\
\hline$\lambda_{111} * 10^{3}$ & -4.1 & -3.6 & -3.0 & -2.4 & -2.1 & -1.9 \\
$\lambda_{100} * 10^{3}$ & -0.25 & -0.15 & -0.11 & - & - & - \\
\hline
\end{tabular}

magnetostrictive $\mathrm{SmFe}_{2}$ compound (the $\mathrm{C} 15$ structure) in the tempera-

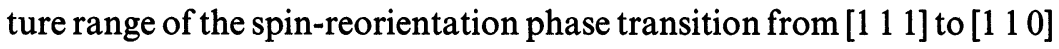
easy direction (ED) (Gaviko et al., 1995).

The substantial role in the texture formation is expected to belong to atoms of hydrogen that are always present in a small amount in the compounds of such type. Since magnetostriction constants $\lambda_{111}$ and $\lambda_{100}$ are negative (see Table I), the lattice is compressed along the crystallographic axis, along which the spontaneous magnetization vector $M_{\mathrm{s}}$ is aligned. Such a spontaneous rhombohedral magnetoelastic lattice deformation starts the hydrogen ordering (Korolyov et al., 1990; Gaviko et al., 1990; 1995).

This in turn results in a sharp increase of the rhombohedral distortion and the occurrence of a local uniaxial magnetic anisotropy, which is added to the initial cubic anisotropy. The ED of the induced magnetic anisotropy is determined by the direction of the magnetoelastic deformation that can be changed by varying the direction of the applied magnetic field at the thermomagnetic treatment. Without thermomagnetic treatment the hydrogenation does not result in a macroscopic anisotropy (Korolyov et al., 1993). In the present paper, the thermal expansion in $\mathrm{SmFe}_{2}$ compound with the magnetic texture has been studied. We observed both compression and an elongation in the same specimen with the magnetic texture at the spin-reorientation transition temperature.

\section{EXPERIMENTAL}

Polycrystalline samples were obtained by melting constituents from 99.9\% $\mathrm{Sm}$ and $99.99 \% \mathrm{Fe}$ in an induction furnace in a purified argon atmosphere and then anneled for $70 \mathrm{~h}$ at $1000 \mathrm{~K}$. After anneling, the amount of secondary phases was not more than $3 \%$. Two types of 
samples have been investigated: bulk samples and samples prepared by powder metallurgical technology. A thermomagnetic treatment of the specimens was done along the axis of the cylindrical specimen and perpendicular to it. Thus, we may have the same specimen with a magnetic texture in the first case for thermomagnetic treatment by magnetic field along the axis of the cylindrical specimen (TMTA). In the second case the thermomagnetic treatment was perpendicular to the axis of the cylindrical specimen (TMTP). The thermal expansion coefficient (TEC) was measured along the axis of the cylindrical specimen using a capacitance dilatometer. The error in measurements of TEC at all temperatures was less than $5 * 10^{-8} \mathrm{~K}^{-1}$ at the specimen length of about $30 \mathrm{~mm}$.

\section{RESULTS AND DISCUSSION}

Temperature dependencies of TEC, $\alpha(T)$, were studied in the temperature range $4.2-300 \mathrm{~K}$ in $\mathrm{SmFe}_{2}$ ferromagnetic compound having spinreorientation phase transition near $190 \mathrm{~K}$ and the Curie temperature near $660 \mathrm{~K}$. The temperature dependences of the TECs of $\mathrm{SmFe}_{2}$ with TMTA and TMTP magnetic texture are shown in Fig. 1. It was found the occurrence of an additional contribution to TEC $\alpha_{\text {extra }}(T)$ relative to the non-magnetic state, which may be described by the DebyeGrüneisen function $\alpha_{\text {lattice }}(T)$ as

$$
\alpha_{\text {extra }}(T)=\alpha(T)-\alpha_{\text {lattice }}(T) .
$$

As illustrated in Fig. 1, this extra contribution to $\alpha_{\text {extra }}(T)$ has the shape of a smoothed $\delta$-function and may be both positive and negative. This phenomenon is observed both in the bulk and in the powdered specimens. The location of the $\delta$-type anomaly is inside the spin-reorientation range. The results are completely reproducible when the thermomagnetic prehistory is repeated carefully. We observed a temperature hysteresis for these anomalies. It is about $2 \mathrm{~K}$, concluding from the position of the extremum values of $\alpha_{\text {extra }}(T)$. These data favor the view that the phase transition is a first order type one (Gaviko et al., 1995).

We believe that the observed $\delta$-type anomalies of the TEC are caused by the magnetic texture of the specimens. The obtained results are understood in the model of domain structure changing and the formation of a new magnetic alignment after spin-reorientation (Korolyov 


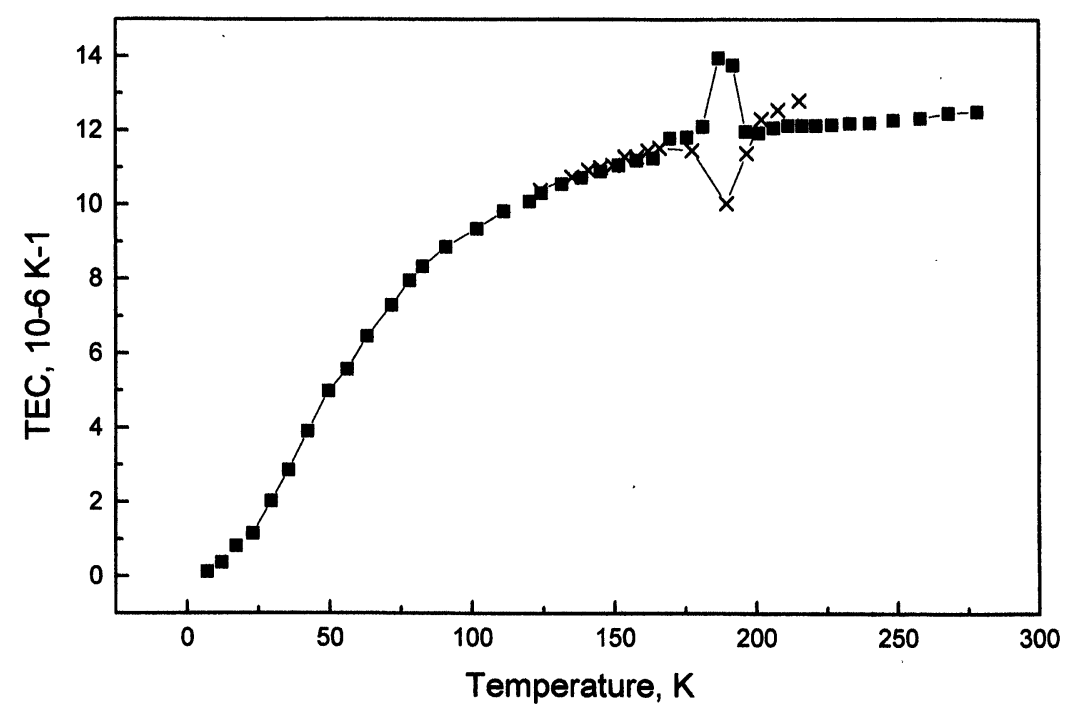

FIGURE 1 Typical temperature dependences of TEC for $\mathrm{SmFe}_{2}$ specimens after thermomagnetic treatment. The direction of the magnetic field at thermomagnetic treatment is along the axis of the specimen $(x)$, and that perpendicular to it $(\boldsymbol{\square})$. For the powder sintered cylindrical specimen, the observation direction is along the axis of the cylindrical specimen.

et al., 1990; Mushnikov et al., 1991). The substantial role in the texture formation is expected to belong to atoms of hydrogen, which occupy e-type $\left(\mathrm{AB}_{3}\right.$-type) interstitial sites (Mushnikov et al., 1995). The phenomenon of anomalies of TEC can be explained as follows. For a domain the elongation observed in the direction of $\left(\beta_{1}, \beta_{2}, \beta_{3}\right)$ can be expressed as

$$
\begin{aligned}
(\delta L / L)= & (3 / 2) \lambda_{100}\left(\alpha_{1}^{2} \beta_{1}^{2}+\alpha_{2}^{2} \beta_{2}^{2}+\alpha_{3}^{2} \beta_{3}^{2}\right) \\
& +3 \lambda_{111}\left(\alpha_{1} \alpha_{2} \beta_{1} \beta_{2}+\alpha_{2} \alpha_{3} \beta_{2} \beta_{3}+\alpha_{3} \alpha_{1} \beta_{3} \beta_{1}\right),
\end{aligned}
$$

where $\left(\alpha_{1}, \alpha_{2}, \alpha_{3}\right)$ are the direction cosines of internal magnetization. The direction of the observation and the internal magnetization at spin-reorientation phase transition can be determined by change in $\left(\alpha_{1}, \alpha_{2}, \alpha_{3}\right)$ and $\left(\beta_{1}, \beta_{2}, \beta_{3}\right)$ under the restrictions

$$
\alpha_{1}^{2}+\alpha_{2}^{2}+\alpha_{3}^{2}=1 \text { and } \beta_{1}^{2}+\beta_{2}^{2}+\beta_{3}^{2}=1 .
$$


Assuming that for the specimen with the magnetic texture TMTA at the transition [ [lll 111$]$ to [llll 110$]$, we have

$$
\begin{array}{ll}
\beta_{1}=\beta_{2}=\beta_{3}=1 / \sqrt{3}, & \\
\alpha_{1}=\alpha_{2}=\alpha_{3}=1 / \sqrt{3} & \text { at }\left[\begin{array}{lll}
1 & 1 & 1
\end{array}\right] \text { phase, } \\
\alpha_{1}=\alpha_{2}=1 / \sqrt{2}, \quad \alpha_{3}=0 & \text { at }\left[\begin{array}{lll}
1 & 1 & 0
\end{array}\right] \text { phase. }
\end{array}
$$

The estimation for the domain elongation at spin-reorientation is given by

$$
(\delta L / L)_{[111]-[110]}=(\delta L / L)_{[111]}-(\delta L / L)_{[110]}
$$

Putting (4) into (5) using (2), we have

$$
(\delta L / L)_{[111]-[110]}=\lambda_{111}-(1 / 2) \lambda_{111}
$$

Similar expressions can be obtained for a domain with the TMTP magnetic texture at the transition [ $\left[\begin{array}{lll}1 & 1 & 1\end{array}\right]$ to $\left[\begin{array}{lll}1 & 1 & 0\end{array}\right]$. For example if

$$
\begin{aligned}
& \beta_{1}=\beta_{2}=1 / \sqrt{6}, \quad \beta_{3}=-\sqrt{(2 / 3)}, \\
& \alpha_{1}=\alpha_{2}=\alpha_{3}=1 / \sqrt{3} \quad \text { at [ }\left[\begin{array}{lll}
1 & 1
\end{array}\right] \text { phase, } \\
& \alpha_{1}=\alpha_{2}=1 / \sqrt{2}, \quad \alpha_{3}=0 \quad \text { at }\left[\begin{array}{lll}
1 & 1 & 0
\end{array}\right] \text { phase. }
\end{aligned}
$$

Putting (7) into (5) using (2), we have

$$
(\delta L / L)_{[111]-[110]}=-(1 / 2) \lambda_{111}-(1 / 4) \lambda_{111}-(1 / 8) \lambda_{100} .
$$

Since for estimation we have

$$
\alpha_{\mathrm{extra}}(T)=(\mathrm{d} / \mathrm{d} T)(\delta L / L)_{[111]-[110]}
$$

So, from these data $\alpha_{\text {extra }}$ is negative $\left(\alpha_{\text {extra }}<0\right)$ in the first case when the observation is along the [1 111$]$ axis, and in the second case $\alpha_{\text {extra }}$ is positive $\left(\alpha_{\text {extra }}>0\right)$. These simple model calculations will allow an understanding of the role of the magnetic texture in the $\alpha(T)$ dependencies of the investigated specimens of $\mathrm{SmFe}_{2}$. 


\section{CONCLUSION}

The spontaneous deformation of the high magnetostrictive polycrystalline specimens at the spin-reorientation phase transition will be determined by the magnetic texture. Because of this the sign of the additional contribution to TEC of $\mathrm{SmFe}_{2}$ compound may be positive or negative according to the direction of the magnetic texture and the direction of the observation.

\section{References}

Gaviko, V.S., Korolyov, A.V. and Mushnikov, N.V. (1990) Magnetostriction-initiated ordering of hydrogen in $\mathrm{Sm}(\mathrm{Fe}, \mathrm{Co})_{2}$ alloys, Journal of Less-Common Metals, 167(2), $119-125$.

Gaviko, V.S., Korolyov, V. and Mushnikov, N.V. (1995) Spontaneous magnetoelastic deformations of the crystal lattice at the spin reorientation temperature range in $\mathrm{SmFe}_{2}$ compound. Solid State Physics (Russia), 37(11), 3241-3247.

Korolyov, A.V., Mushnikov, N.V., Andreev, A.V. and Gaviko, V.S. (1990) Magnetic and magnetoelastic properties in $\mathrm{Sm}\left(\mathrm{Fe}_{1-x} \mathrm{Co}_{x}\right)_{2}$ intermetallics compounds. Physics of Metals and Metallography, No. 2, 52-57.

Korolyov, A.V., Gaviko, V.S. and Mushnikov, N.V. (1993) Quasi-magnetoelastic after effect and induced magnetic anisotropy caused by absorbed hydrogen in rare-earth $\mathrm{MgCu}_{2}$-structure compounds. IEEE Trans. Magn., 29(6), 2899-2901.

Mushnikov, N.V., Korolyov, A.V., Gaviko, V.S., Raevski, Ye.I. and Pareti, L. (1991) Induced magnetic anisotropy in $\mathrm{Sm}(\mathrm{Fe}, \mathrm{Co})_{2}$ compounds. J. Appl. Phys., 70(5), 27682773, 3241-3247.

Mushnikov, N.V., Gaviko, V.S., Korolyov, A.V. and Zajkov, N.K. (1995) Hydrogeninduced magnetic anisotropy and crystal lattice distortion in $\mathrm{Sm}_{1-x} \mathrm{~Tb}_{x}\left(\mathrm{Fe}_{0.2} \mathrm{Co}_{0.8}\right)_{2}$ compounds. Journal of Alloys and Compounds, 218, 165-172. 